\title{
NUMERICAL SIMULATIONS OF LOW REYNOLDS NUMBER FLOWS PAST ELASTICALLY MOUNTED CYLINDER
}

\author{
R. A. Gonçalves a, \\ P. R. F. Teixeira ${ }^{b}$, \\ and E. Didier ${ }^{\text {c, d }}$ \\ ${ }^{a, b}$ Universidade Federal do Rio Grande \\ Escola de Engenharia \\ Campus Carreiros \\ CP. 96201-900, Rio Grande, Rio Grande do \\ Sul, Brazil \\ a rafa.a.g@ibest.com.br \\ b pauloteixeira@furg.br \\ ${ }^{\mathrm{c}}$ Laboratório Nacional de Engenharia Civil \\ Departamento de Hidráulica e Ambiente \\ Av. do Brasil, 101, 1700-066, Lisboa, Portugal \\ edidier@Inec.pt \\ ${ }^{\mathrm{d}}$ Universidade Nova de Lisboa \\ Faculdade de Ciências e Tecnologia \\ Campus de Caparica \\ 2829-516, Monte de Caparica, Portugal
}

\section{ABSTRACT}

The vortex-induced vibration (VIV) phenomenon has drawn the attention of researchers in Engineering for several decades. An example is the riser used for petroleum exploration, in which it is subjected to marine flows that may cause oscillations due to vortex shedding. In this paper, numerical analyses of the phenomena that occur in the interaction among flows at low Reynolds numbers and elastically mounted cylinders are presented. The simulation is carried out by using the numerical model Ifeinco that uses a semi-implicit two-step Taylor-Galerkin method to discretize the Navier-Stokes equations and the arbitrary Lagrangean-Eulerian formulation to follow the cylinder motion. The rigid body motion description is calculated by using the Newmark method. Firstly, the characteristics of the vortex generation process for the fixed cylinder are analyzed. In this case, the Strouhal number, the mean drag and the RMS lift coefficients for Reynolds numbers ranging from 90 to 140 are shown. Afterwards, an analysis of a flexible supported cylinder (with a spring and a damper) in transverse direction subject to flows with Reynolds numbers ranging from 90 to 140 is carried out. The cylinder displacement and the vibration frequencies are studied; the synchronization between the vortex shedding and the vibration frequency (lock-in) is analyzed. Similar results to the experimental ones developed by Anagnostopoulos and Bearman (1992) were obtained in this study.

Keywords: finite element method, oscillating cylinder, fluid-structure interaction.

\section{NOMENCLATURE}

c Damping coefficient, $\mathrm{kg} / \mathrm{s}$

$\mathrm{C}_{\mathrm{D}} \quad$ Drag coefficient

$\mathrm{C}_{\mathrm{L}} \quad$ Lift coefficient

$\mathrm{D} \quad$ Diameter, $\mathrm{m}$

F Force, N

$f \quad$ Frequency, $\mathrm{Hz}$

$\mathrm{F}_{\mathrm{D}} \quad$ Drag force, $\mathrm{N}$

$\mathrm{F}_{\mathrm{L}} \quad$ Lift force, $\mathrm{N}$

$f_{n} \quad$ Natural frequency, $\mathrm{Hz}$

$g$ Gravity acceleration, $\mathrm{m} / \mathrm{s}^{2}$

$m$ mass, $\mathrm{kg}$

$p \quad$ Pressure, $\mathrm{Pa}$

Re Reynolds number

St Strouhal number

$U$ Momentum per volume, $\mathrm{kg} \mathrm{m} / \mathrm{s} / \mathrm{m}^{3}$

$U_{\infty} \quad$ Free stream velocity, $\mathrm{m} / \mathrm{s}$

v Fluid velocity, $\mathrm{m} / \mathrm{s}$

w Mesh velocity, $\mathrm{m} / \mathrm{s}$

$\mathrm{Y} \quad$ Amplitude of cylinder oscillation, $\mathrm{m}$

$y \quad$ Cylinder displacement, $\mathrm{m}$

$\dot{y} \quad$ Cylinder Velocity, $\mathrm{m} / \mathrm{s}$

$\ddot{y} \quad$ Cylinder acceleration, $\mathrm{m} / \mathrm{s}^{2}$

\section{Greek symbols}

$\tau \quad$ Viscous stress tensor, $\mathrm{Pa}$

$\rho \quad$ Specific mass, $\mathrm{kg} / \mathrm{m}^{3}$

\section{$k \quad$ Spring stiffness, $\mathrm{N} / \mathrm{m}$ \\ $\mu \quad$ Viscosity, $\mathrm{kg} /(\mathrm{ms})$}

\section{INTRODUCTION}

Vortex-induced vibration (VIV) is a phenomenon that is found in several engineering fields. Some examples are the following: wind can cause oscillations on bridges, slender buildings, chimneys and energy transmission cables; flows with high velocities can induce orbital movements in internal tubes of a heat exchanger; and currents and waves can cause vibration on pipelines.

The wake around a circular cylinder due to a uniform flow leads to variety of complex phenomena. Despite the simplicity of geometry, the flow around a cylinder is very complicated and of particular importance, since it may induce unsteady forces on structures associated with vortex shedding. Therefore, this case has been studied for several decades, and, nowadays, the behavior of the flow is known. For Reynolds number (Re) up to 49, two symmetric stationary recirculation zones attached to the rear part of the cylinder wall are observed. From 49 to 190 , the wake is still laminar and twodimensional and it is composed by two periodic staggered rows of alternating vortices (von Kármán vortex shedding). For higher Reynolds numbers (from 190 to 260), the wake becomes threedimensional and progressively turbulent. This regime 
is followed by a shear layer transition (up to 1200), in which separating shear layers become unstable, and, finally, by the boundary layer transition (around $10^{5}$ ) associated with fast decrease of the drag coefficient. For these regimes, the flow exhibits a periodicity which is known as Strouhal frequency. When a periodic vortex street is well established, this frequency corresponds to that of the vortex shedding frequency; in other cases, in which the von Kármán streets are not clearly visible, the frequency can be defined as the one of the fluctuations of the streamwise velocity component, for example (Placzek et al., 2009).

In many applications, the cylinder oscillates and interacts with the vortex shedding process. For forced oscillations in a range of frequency and amplitude, the cylinder motion is able to control the instability mechanism generated by vortex shedding. One of the most interesting characteristics of this fluid-structure interaction is the synchronization (lock-in) between the vortex shedding and the vibration frequency. Similar phenomena are observed for VIV, in which the flow causes the oscillation of the cylinder at its natural frequency. This frequency depends on the mass, the rigidity and the damping of the cylinder. In this phenomenon, which occurs in a range of flow velocity, the amplitude reaches a maximum value.

This complex fluid-structure interaction phenomenon is still a good test case to validate the numerical models. Several numerical analyses can be found in the literature for a large range of Reynolds numbers, including Reynolds Averaged NavierStokes (RANS) methods (Saghafian et al., 2003; Guilmineau and Queutey, 2004), Large Eddy Simulations (LES) (Breuer, 2000; Pasquetti, 2005; Al-Jamal and Dalton, 2004), Direct Numerical Simulations (DNS) and methods that use finite volume or finite element approximations to solve the Navier-Stokes equations (Anagnostopoulos and Bearman, 1992; Nobari and Naredan, 2006; Mittal and Kumar, 2001).

This paper describes simulations which are carried out by using the numerical model Ifeinco (Teixeira and Awruch, 2005) that uses a semiimplicit two-step Taylor-Galerkin method to discretize the Navier-Stokes equations and the arbitrary Lagrangean-Eulerian formulation to follow the cylinder motion. The rigid body motion description is calculated by using the Newmark method. Firstly, the characteristics of the vortex generation process for the fixed cylinder are analyzed. In this case, the Strouhal number, the mean drag and the RMS lift coefficients for Reynolds numbers ranging from 90 to 140 are shown and compared with experimental and numerical results of the literature. Afterwards, an analysis of a flexible supported cylinder (with a spring and a damper) in transverse direction subject to flows with Reynolds numbers ranging from 90 to 140 is carried out. The cylinder displacement and the vibration frequencies are studied and the synchronization between the vortex shedding and the vibration frequency (lock-in) is analyzed. Numerical results are similar to experimental ones obtained by Anagnostopoulos and Bearman (1992).

\section{NUMERICAL MODEL}

The numerical model Ifeinco is based on a partitioned scheme, in which the fluid flow and the structure are solved in two-way interaction. Basically, the fluid-structure interaction adopted by the code consists in the following steps: (a) update the variables of the flow from instant $t$ to $t+\Delta t$; (b) impose pressure and viscous stress as a load to the structure; (c) update the variables of the structure from instant $t$ to $t+\Delta t$; (d) impose the body motion to the flow in terms of the updated velocity vector and boundary position.

Basically, updating the variables of the flow consists of following steps (Teixeira and Awruch, 2005):

a) Calculate non-corrected velocity $\tilde{U}_{i}$ at $t+\Delta t / 2$, where the pressure term is at $t$ instant, according to the equation:

$$
\begin{aligned}
& \widetilde{U}_{i}^{n+1 / 2}=U_{i}^{n}- \\
& -\frac{\Delta t}{2}\left(\frac{\partial f_{i j}^{n}}{\partial x_{j}}-\frac{\partial \tau_{i j}^{n}}{\partial x_{j}}+\frac{\partial p^{n}}{\partial x_{i}}-\rho g_{i}-w_{j}^{n} \frac{\partial U_{i}^{n}}{\partial x_{i}}\right)
\end{aligned}
$$

where $\rho$ is the specific mass, $p$ is the pressure, $g_{i}$ are the gravity acceleration components, $\mathrm{V}_{i}$ are the velocity components, $w_{i}$ are the velocity components of the reference system and $\tau_{i j}$ is the viscous stress tensor $U_{i}=\rho_{\mathrm{v}_{i}}, f_{i j}=\mathrm{v}_{j}\left(\rho \mathrm{v}_{i}\right)=\mathrm{v}_{j} U_{i}(i, j=1,2)$.

b) Update the pressure $p$ at $t+\Delta t$, given by the Poisson equation:

$$
\frac{1}{c^{2}} \Delta p=-\Delta t\left[\frac{\partial \widetilde{U}_{i}^{n+1 / 2}}{\partial x_{i}}-\frac{\Delta t}{4} \frac{\partial}{\partial x_{i}} \frac{\partial \Delta p}{\partial x_{i}}\right]
$$

where $\Delta p=p^{n+1}-p^{n}$ and $i=1,2$.

c) Correct the velocity at $t+\Delta t / 2$, adding the pressure variation term from $t$ to $t+\Delta \mathrm{t} / 2$, according to the equation:

$U_{i}^{n+1 / 2}=\widetilde{U}_{i}^{n+1 / 2}-\frac{\Delta t}{4} \frac{\partial \Delta p}{\partial x_{i}}$

d) Calculate the velocity at $t+\Delta t$ using variables updated in the previous steps as follows:

$$
\begin{gathered}
U_{i}^{n+1}=U_{i}^{n}-\Delta t\left(\frac{\partial f_{i j}^{n+1 / 2}}{\partial x_{j}}-\frac{\partial \tau_{i j}^{n+1 / 2}}{\partial x_{j}}+\right. \\
\left.+\frac{\partial p^{n+1 / 2}}{\partial x_{i}}-w_{j}^{n+1 / 2} \frac{\partial U_{i}^{n+1 / 2}}{\partial x_{i}}-\rho g_{i}\right)
\end{gathered}
$$


The classical Galerkin weighted residual method is applied to the space discretization of Eq. (1), (2), (3) and (4), and a triangular element is employed. In the variables at $t+\Delta t / 2$ instant, a constant shape function is used, and in the variables at $t$ and $t+\Delta t$, a linear shape function is employed (Teixeira and Awruch, 2001). The mesh velocity vertical component $w_{2}$ is computed to diminish element distortions, keeping prescribed velocities on moving and stationary boundary surfaces. The mesh movement algorithm adopted in this paper uses a smoothing procedure for the velocities based on these boundary lines. The updating of the mesh velocity at node $i$ of the finite element domain is based on the mesh velocity of the nodes $j$ that belong to the boundary lines.

In order to update the rigid-body motion structure, it is necessary to calculate displacements and rotations of a hypothetical concentrated mass at its gravity center. In this study case, there is only movement in transverse direction (one degree of freedom - DOF) and, consequently, displacement, velocity and acceleration in this direction are the variables to be determined at each time step. To update the variables of the structure, the rigid motion of the cylinder is calculated at each instant, after the variables of the flow (pressure and viscous stress) are known. For this study case, one DOF dynamic equation is considered for the transverse direction, as follows:

$m \ddot{y}+c \dot{y}+k y=F$

where $\ddot{y}, \dot{y}$ and $y$ are the transverse acceleration, velocity and displacement, respectively; $m$ is the mass; $c$ is the damping coefficient; $k$ is the stiffness; and $F$ is the dynamic force. In this code, Eq. (5) is discretized in time by using the implicit Newmark method (Bathe, 1996) and the acceleration, the velocity and the displacement in transverse direction are calculated at each time step.

\section{NUMERICAL SIMULATIONS}

The case study consists of a cylinder (diameter and mass equal to $0.0016 \mathrm{~m}$ and $0.2979 \mathrm{~kg}$, respectively) subject to a uniform water flow (specific mass, $\rho$, and viscosity, $\mu$, equal to $1000.0 \mathrm{~kg} / \mathrm{m}^{3}$ and $0.001 \mathrm{~kg} /(\mathrm{ms})$, respectively). The cylinder is mounted on a spring and a damper in transverse direction and fixed towards the flow. The spring stiffness, $k$, is equal to $579 \mathrm{~N} / \mathrm{m}$ and the damping coefficient, $c$, is equal to $0.0325 \mathrm{~kg} / \mathrm{s}$. The natural frequency of this system is $f n=7.016 \mathrm{~Hz}$ (Anagnostopoulos and Bearman, 1992).

The influence of the size of the computational domain was analyzed for Reynolds number equal to 135. The best computational domain that satisfied both accuracy and computational cost criteria was a rectangle $0.320 \mathrm{~m}$ wide and $0.384 \mathrm{~m}$ long, as shown in Fig. 1. The cylinder center is located at the center of the domain in transverse direction to the flow and $0.160 \mathrm{~m}$ from its left side in longitudinal direction. In this computational domain, the smallest distance from the boundary to the cylinder center is equal to 100D. After analyzing the mesh convergence, a finite element unstructured mesh composed by triangles with 200 elements around the cylinder was used. Element sizes increase gradually towards the boundaries of the domain. The mesh has 298143 nodes and 595526 elements. A constant velocity is imposed on the left side of the computational domain; on the lateral boundaries, a slide condition is imposed; and the right side is free exit, but null pressure is imposed on its middle. The time step used for the simulations is equal to $5.0 \times 10^{-5} \mathrm{~s}$.

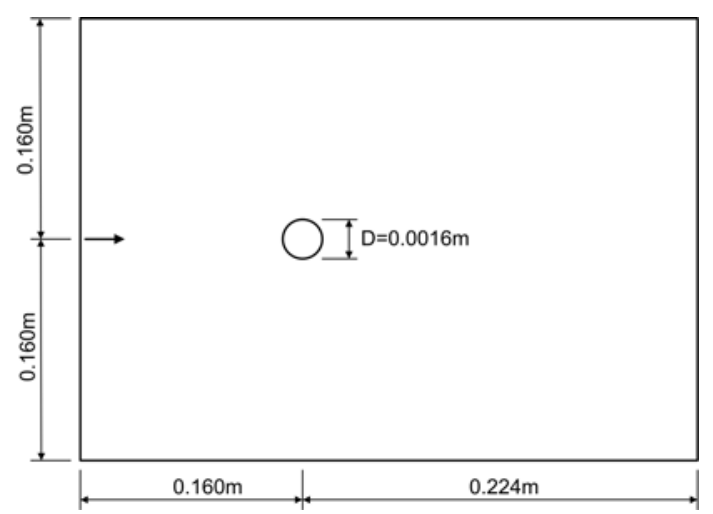

Figure 1. Numerical domain.

First, the behavior of the flow considering the fixed cylinder is analyzed. Specifically, the flow characteristics at $\mathrm{Re}=105$ (velocity $U_{\infty}$ equal to $0.065625 \mathrm{~m} / \mathrm{s}$ ) are studied. Figure 2 shows the velocity vectors and streamlines at eight instants along one period of vortex formation, respectively. The vortex formation was clearly observed, showing two different regions behind the cylinder where the flow separation occurs. Near the cylinder surface, while the larger vortex is in one direction, the opposing vortex is in another one.

Drag $\left(\mathrm{F}_{\mathrm{D}}\right)$ and lift $\left(\mathrm{F}_{\mathrm{L}}\right)$ forces on the cylinder obtained by numerical simulation for $\mathrm{Re}=105$ are shown in Fig. 3. The drag force has a periodic behavior with a little variation around $0.0045 \mathrm{~N}$, whereas the lift force has a periodic behavior with amplitude equal to $0.0012 \mathrm{~N}$ and frequency equal to $6.828 \mathrm{~Hz}$

Figure 4 shows a comparison among the Strouhal numbers, $\mathrm{St}=f \mathrm{D} / U_{\infty}$, obtained by numerical results (where $f$ is equal to the lift force frequency) for Re from 90 to 140 and experimental results of Willianson (Willianson, 1989). Numerical results are in very good agreement with experimental ones; the mean difference was only $0.03 \%$.

In Fig. 5, a comparison of the mean drag coefficient $\left(\mathrm{C}_{\mathrm{D}}\right)$ with numerical ones obtained by Poldsziech and Grundmann (2007) for the same range of the Re is shown. The authors used a Spectral 
Element Method and presented two curves with sizes of the computational domain of 70D and 4000D. It may be noticed that the curve of this study is located between both Poldsziech and Grundmann's curves and the mean differences related to the curves are only $0.8 \%(4000 \mathrm{D})$ and $0.4 \%$ (70D).

The root mean square (RMS) lift coefficient $\left(\mathrm{C}_{\mathrm{L}}\right)$ is shown in Fig. 6 and it is compared with numerical one obtained by Baranyi and Lewis (2006), who used a Grid Based Method and a size of the computational domain of 40D. In this case, the mean difference between both numerical results is only $0.2 \%$.

It is worth mentioning that the high accuracy of the parameters $S t$ and $C_{L}$ shows the capacity of the numerical model Ifeinco to reproduce the frequency and the magnitude of forces that is imposed over the circular cylinder in the fluid-structure interaction process.

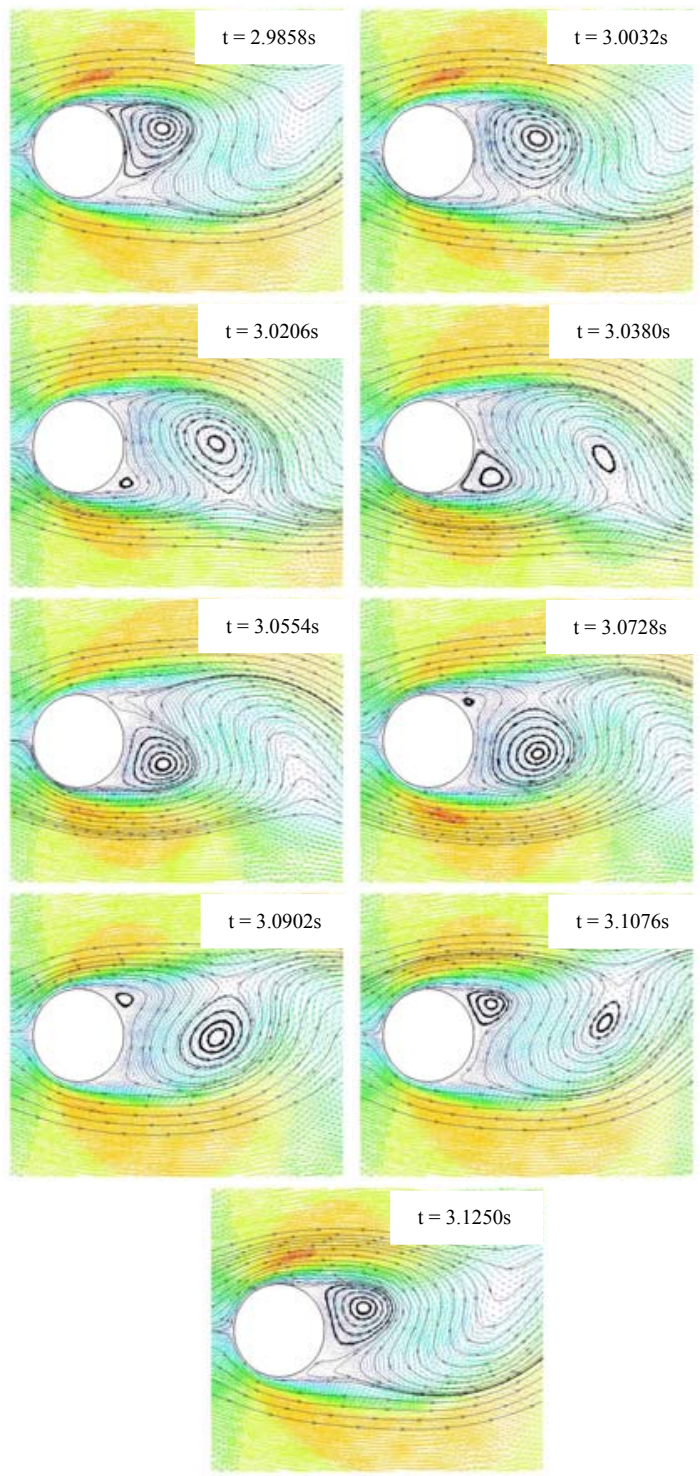

Figure 2. Velocity vectors at eight instants along a period of vortex formation.

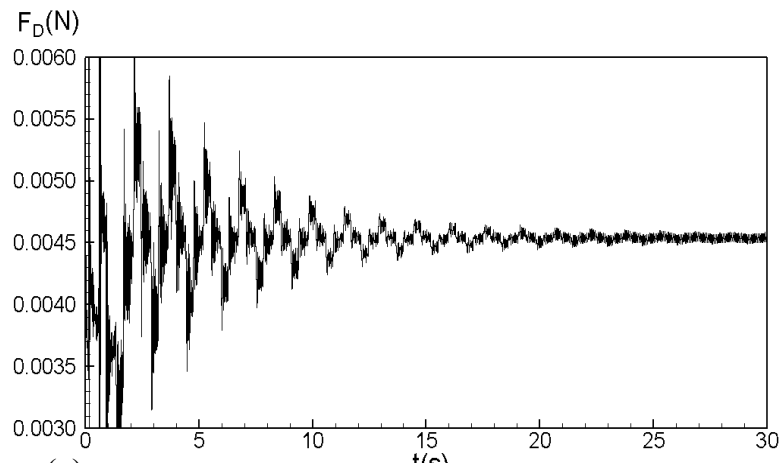

(a)

$t(s)$

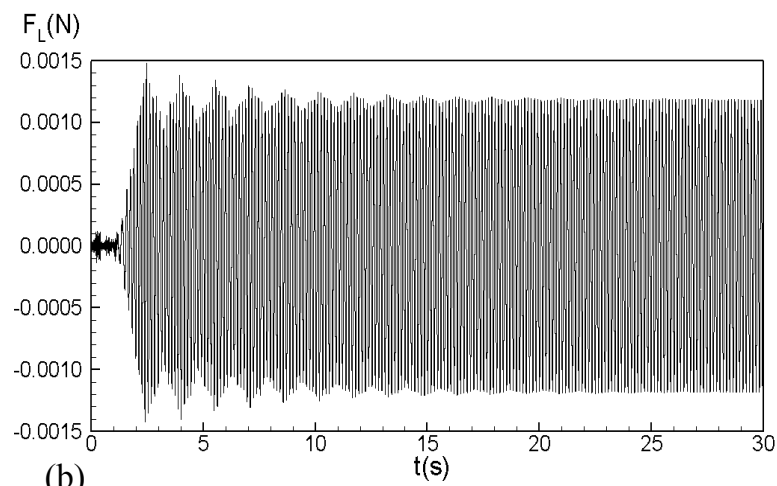

(b)

Figure 3. Drag (a) and lift (b) forces for fixed cylinder with $\mathrm{Re}=105$.

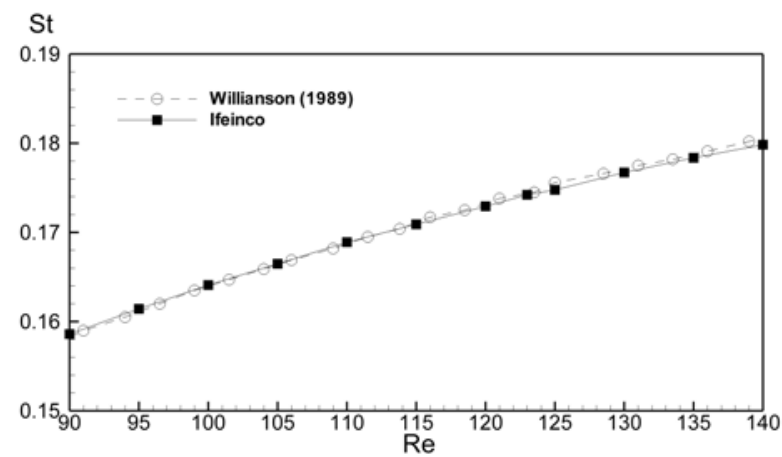

Figure 4. Strouhal numbers versus Reynolds numbers.

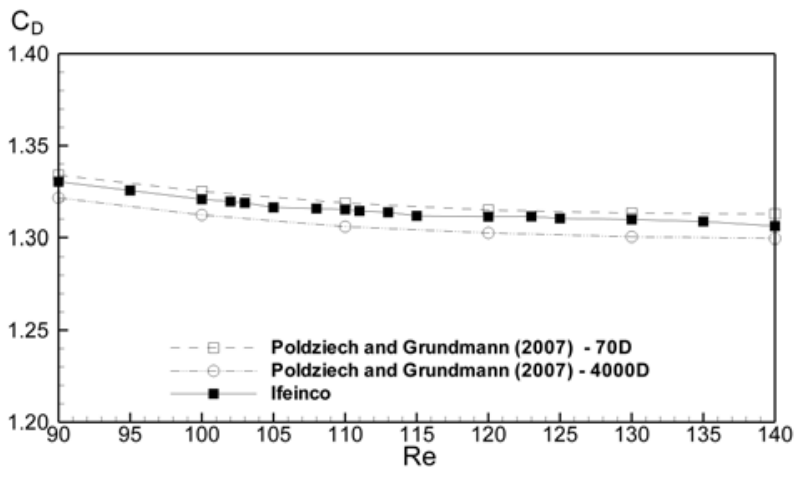

Figure 5. Mean drag coefficient $\left(\mathrm{C}_{\mathrm{D}}\right)$ versus Reynolds numbers. 


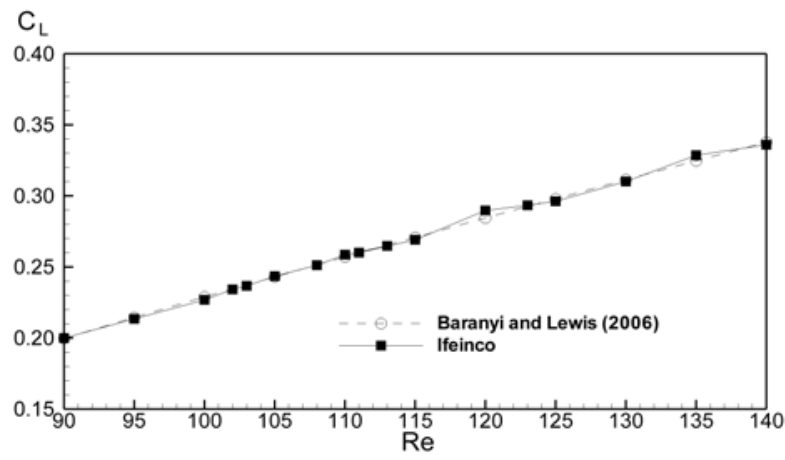

Figure 6. RMS lift coefficient $\left(\mathrm{C}_{\mathrm{L}}\right)$ versus Reynolds numbers.

Afterwards, the interaction among a cylinder mounted on an elastic fixing in transverse direction and flows at Reynolds numbers from 90 to 140 is analyzed.

Figure 7 shows the relation between the amplitude (Y) of the cylinder oscillation and its diameter (D) in function of Reynolds numbers (90 to 140). Figure 8 shows the relation between the frequency of vibration (obtained by the time series of the $\left.C_{L}\right)$ and the natural frequency $(f / f n)$ in function of Reynolds numbers.

The numerical results show that the lock-in phenomenon was captured for Reynolds numbers between 102 and 113. This is observed due to the increase of the amplitude of cylinder motion and the equality of vibration and natural frequencies. Out of the lock-in region, the amplitudes of cylinder motion are negligible and the vibration frequencies are similar to that obtained for a fixed cylinder, described by the Willianson's curve.

Figures 7 and 8 also show the results obtained experimentally by Anagnostopoulos and Bearman (1992) and numerically by Dettmer and Perić (2006). The latter used a model that employs the stabilized low order velocity-pressure finite elements, an arbitrary Lagrangian-Eulerian formulation and the discrete implicit generalized- $\alpha$ method for the rigid body motion. Both numerical results are similar in terms of amplitudes and frequencies along the Reynolds number range. The numerical amplitude values are always smaller than experimental ones and there are little differences in the lock-in range. The frequencies obtained numerically show the same behavior: in the lock-in region, the vibration frequency is equal to the natural frequency, while, out of the lock-in, the vibration frequency follows the Strouhal ones. The differences among amplitudes obtained experimentally and numerically in the Reynolds number range in the lock-in region were explained by Dettmer and Peric (2006) comparing the numerical domain and boundary conditions and the real situation of the experience. The experiment was carried out in a $0.70 \mathrm{~m}$ deep channel where $0.12 \mathrm{~m}$ of the cylinder was submerged. The lack of a horizontal plate in the end of the submerged cylinder allowed the vortex shedding in this region. This fact and the influence of the free surface contribute to develop the three-dimensional behavior of the flow, unlike the numerical conditions.

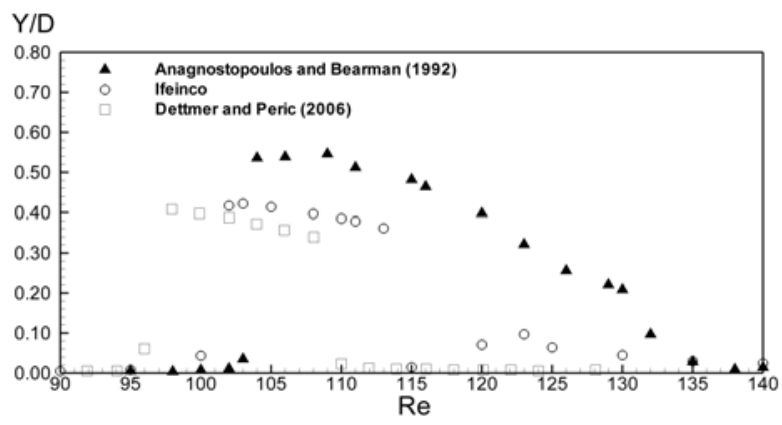

Figure 7. Amplitude (Y/D) versus Reynolds numbers.

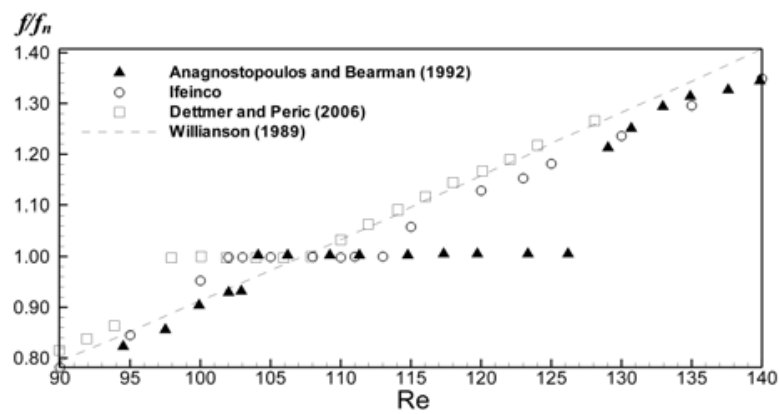

Figure 8. Frequency of vibration $(f / f n)$ versus Reynolds numbers.

Figure 9 shows the behavior of the drag and the lift forces for oscillating and fixed cylinders with $\mathrm{Re}=105$. Unlike the drag force for the fixed cylinder, which has a small harmonic variation around $0.0045 \mathrm{~N}$, the force oscillates between $0.0050 \mathrm{~N}$ and $0.0073 \mathrm{~N}$ at a frequency equal to $14.085 \mathrm{~Hz}$ (almost twofold the frequency in transverse direction) for the oscillating cylinder. In the transverse direction, the lift forces differ in terms of frequency and amplitude. The amplitudes for the fixed and the oscillating cylinders are $0.0012 \mathrm{~N}$ and $0.0014 \mathrm{~N}$ and their frequencies are $6.828 \mathrm{~Hz}$ and $6.984 \mathrm{~Hz}$, respectively. Both frequencies are related to the vortex shedding and the latter is closer to the natural frequency of the dynamic system of the cylinder $(f n=7.016 \mathrm{~Hz})$.

A representative case out of the lock-in region $(\mathrm{Re}=123)$ was chosen to show its different force behavior. Figure 10 shows the time series of the drag and the lift forces for $\mathrm{Re}=123$. The drag forces exhibit little variation around $0.0062 \mathrm{~N}$ for both fixed and oscillating cylinders, with more disturbances in the case of the fixed cylinder. The drag force for the fixed cylinder has a harmonic behavior with amplitude and frequency equal to $0.0020 \mathrm{~N}$ and $8.313 \mathrm{~Hz}$, respectively. For the oscillating cylinder, this force oscillates periodically (frequency of $8.163 \mathrm{~Hz}$ ) within an envelope with minimum and maximum amplitudes of $0.0014 \mathrm{~N}$ and $0.0020 \mathrm{~N}$, 
respectively. Although these amplitudes are higher than those in the previous case $(\mathrm{Re}=105)$, the displacements are smaller, since this Reynolds number, $\mathrm{Re}=123$, is out of the lock-in region.

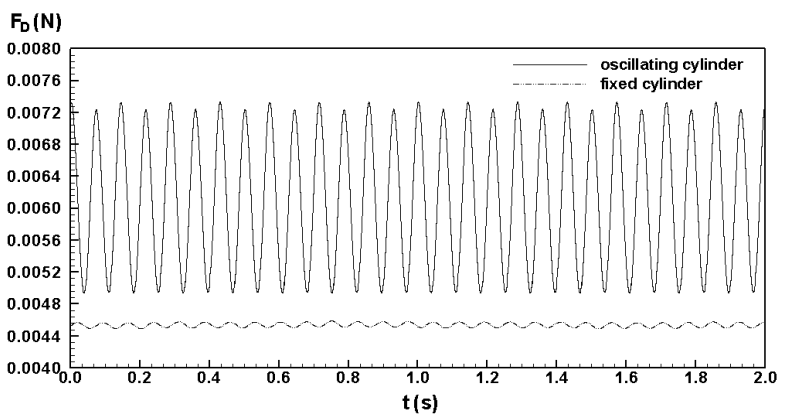

(a)

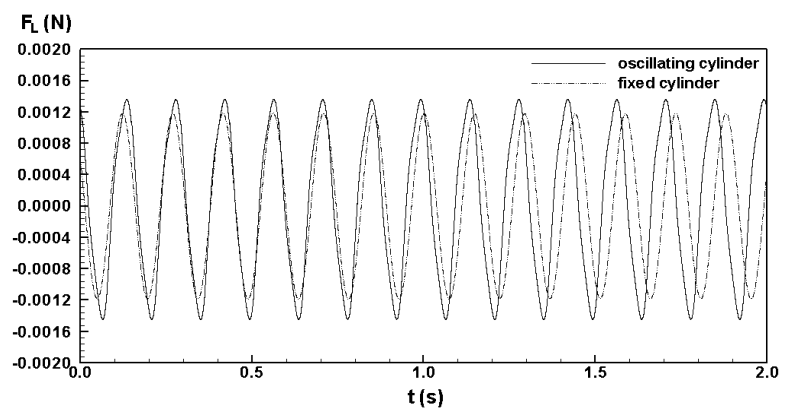

(b)

Figure 9. Drag (a) and lift (b) forces for cylinder with $\mathrm{Re}=105$.
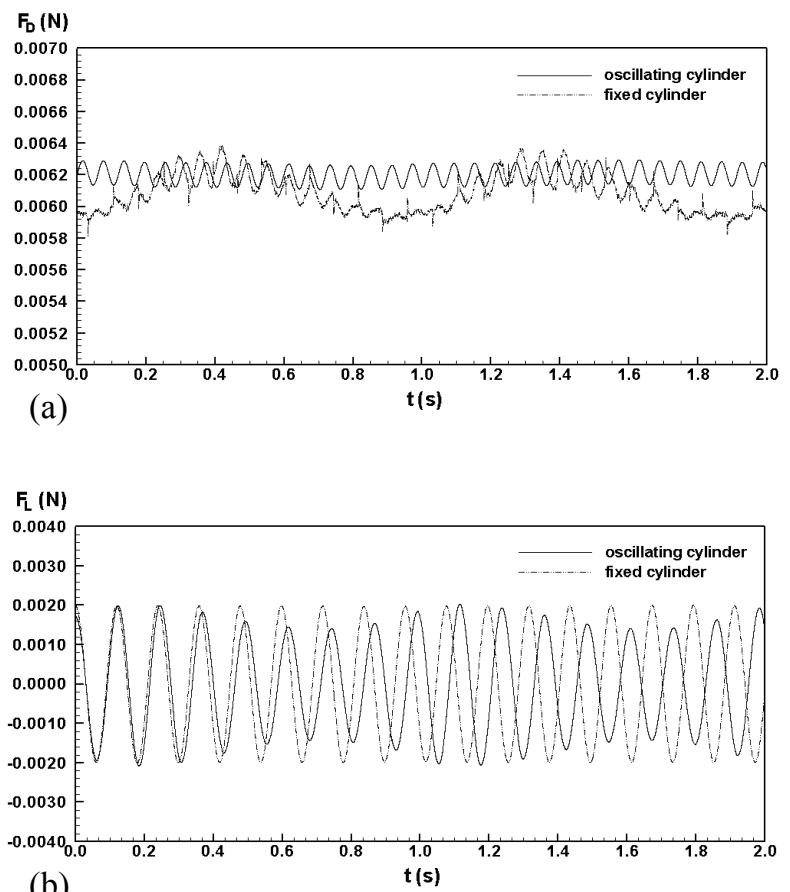

(b)

Figure 10. Drag (a) and lift (b) forces for cylinder with $\mathrm{Re}=123$.

\section{CONCLUSIONS}

In this paper, numerical analyses of the phenomena that occur in the interaction among flows at low Reynolds numbers and elastically mounted cylinders were presented. The simulation was carried out by a numerical model, Ifeinco, that uses a semiimplicit two-step Taylor-Galerkin method to discretize the Navier-Stokes equations and the arbitrary Lagrangean-Eulerian formulation to follow the cylinder motion. The rigid body motion description is calculated by using the Newmark method.

For the fixed cylinder, the behavior of the vortex formation was correctly reproduced: near the cylinder surface, the larger vortex was in one direction, while the opposing vortex was in another one. The Strouhal numbers were calculated for Reynolds number range from 95 to 140 . These values were similar to those obtained by Willianson's experiments. The mean drag and the RMS lift coefficients were also calculated and compared with those obtained by Poldsziech and Grundmann (2007) and Baranyi and Lewis (2006), respectively. Good agreements were obtained $(0.8 \%$ and $0.2 \%$, respectively) and showed the accuracy of the numerical simulation.

For interaction among the cylinder mounted on an elastic fixing in transverse direction and flows, the lock-in phenomenon was captured for Reynolds numbers between 105 and 110 , characterized by the increase of the amplitude and the equality of vibration and natural frequencies. Comparing the numerical and the experimental results (Anagnostopoulos and Bearman, 1992), some differences were observed due to the presence of the three-dimensional effects of the experiment that were not considered in these numerical simulations. Comparing the results obtained by Ifeinco with numerical ones obtained by Dettmer and Perić (2006), it was show that both numerical results are similar in terms of amplitudes of cylinder motion and frequencies for the Reynolds number range studied, confirming the ability of the Ifeinco code for modeling complex fluid-structure interaction phenomena.

\section{ACKNOWLEDGEMENTS}

The first author wishes to thank CAPES for the post-graduate scholarship. The second author acknowledges the support of Conselho Nacional de Desenvolvimento Científico e Tecnológico (CNPq project 303308/2009-5).

\section{REFERENCES}

Al-Jamal, H., and Dalton, C., 2004, Vortex Induced Vibrations Using Large Eddy Simulation at a Moderate Reynolds Number, Journal of Fluids and 
Structures, Vol. 19, No. 1, pp. 73-92.

Anagnostopoulos, P., and Bearman P. W., 1992,

Response Characteristics of a Vortex-Excited Cylinder at Low Reynolds Numbers, Journal of Fluids and Structures, Vol. 14, No. 6, pp. 39-50.

Baranyi, L., and Lewis, R. I., 2006, Comparison of Grid-Based and Vortex Dynamics Predictions of Low Reynolds Number Cylinder Flows, Aeronautical Journal, No. 2983, pp. 63-71.

Bathe, K. J., 1996, Finite Element Procedures, Prentice-Hall.

Breuer, M., 2000, A Challenging Test Case for Large Eddy Simulation: High Reynolds Number Circular Cylinder Flow. International Journal of Heat Fluid Flow, Vol. 21, No. 5, pp. 648-654.

Dettmer, W., and Perić, D., 2006, A Computational Framework for Fluid-Rigid Body Interaction: Finite Element Formulation and Applications, Computer Methods in Applied Mechanics and Engineering, Vol. 195, pp. 16331666.

Guilmineau, E., and Queutey, P., 2004, Numerical Simulation of Vortex-Induced Vibration of a Circular Cylinder with Low Mass-Damping in a Turbulent Flow, Journal of Fluids and Structures, Vol. 19, pp. 449-466.

Mittal, S., and Kumar, V., 2001, Flow-Induced Vibrations of a Light Circular Cylinder at Reynolds Numbers, Journal of Sound and Vibration, Vol. 5, No. 245, pp. 923-946.

Nobari, M. R. H., and Naredan, H., 2006, A Numerical Study of Flow Past a Cylinder with Cross Flow and Inline Oscillation. Computers of Fluids, Vol. 35, No. 4, pp. 393-415.

Pasquetti, R., 2005, High-Order Methods for the Numerical Simulation of Vertical and Turbulent Flows-High-Order LES Modeling of Turbulent Incompressible Flow, Comptes Rendus Mécanique, Vol. 333, No. 1, pp. 39-49.

Poldsziech, O., and Grundmann, R., 2007, A Systematic Approach to the Numerical Calculation of Fundamental Quantities of the Two-Dimensional Flow Over Circular Cylinder, Journal of Fluids and Structures, Vol. 23, pp. 479-499.

Placzek, A., Sigrist, J., and Hamdouni, A., 2009, Numerical Simulation of an Oscillating Cylinder in a Cross-Flow at Low Reynolds Number: Forced and Free Oscillations, Computer \& Fluids, Vol. 38, No. 1, pp. 80-100.

Saghafian M., Stansby, P. K., Saidi, M. S., and Apsley, D. D., 2003, Simulation of Turbulent Flows Around a Circular Cylinder Using Nonlinear EddyViscosity Modeling: Steady and Oscillatory Ambient Flows, Journal of Fluids and Structures, Vol 15, No. 1, pp. 1213-1236.

Teixeira, P. R. F., and Awruch, A. M., 2001, Three-Dimensional Simulation of High Compressible Flows Using a Multi-Time-Step Integration Technique with Subcycles, Applied Mathematical Modelling, Vol. 25, pp. 613-627.
Teixeira, P. R. F., and Awruch, A. M., 2005, Numerical Simulation of Fluid-Structure Interaction Using the Finite Element Method, Computers \& Fluids, Vol. 34, pp. 249-273.

Willianson, C. H. K., 1989, Oblique and Parallel Modes of Vortex Shedding in the Wake of a Circular Cylinder at Low Reynolds Numbers, Journal of Fluid Mechanics, Vol. 206, pp. 579-627.

Received: September 30, 2012

Revised: October 30, 2012

Accepted: November 30, 2012 